\title{
Home-office na produção científica da América Latina e Caribe: evolução e impulso na pandemia de Covid-19
}

\section{Home-office in scientific output of Latin America and Caribbean: evolution and impulse in the Covid-19 pandemic}

\author{
Gonzalo Rubén Alvarez \\ Doutor em Comunicação e Informação \\ Universidade Federal Fluminense \\ gonzalorubenalvarez@gmail.com \\ Dirce Maria Santin \\ Doutora em Comunicação e Informação \\ Universidade Federal do Rio Grande do Sul \\ dirce.santin@ufrgs.br
}

\section{Resumo}

Home-office é um tema relevante no mundo do trabalho e recebeu impulso com a pandemia de Covid-19, que exigiu a adaptação do trabalho ao distanciamento social. Este artigo analisa a produção científica dos países da América Latina e Caribe sobre home-office nas últimas duas décadas. Com abordagem cientométrica, baseada nos dados de artigos indexados na Web of Science (WoS) e SciELO Citation Index (SciELO Cl), analisa a evolução dos estudos, os periódicos de circulação regional e internacional, a distribuição geográfica e temática e a colaboração na coautoria dos artigos. Os resultados mostram um tema em desenvolvimento, com intenso crescimento a partir da pandemia de Covid-19, que combina um enfoque prioritário em periódicos e idiomas locais com a publicação em canais internacionais. Mais explorado nas Ciências Sociais, o assunto registra baixa colaboração internacional e desenvolve-se em torno de temáticas centrais como teletrabalho, saúde, educação e Covid-19. Conclui-se que o impulso recebido pelo assunto na pandemia de Covid-19 tende a ampliar e diversificar as pesquisas e as discussões sobre o home-office como forma de trabalho de caráter provisório, excepcional ou sistemático na sociedade contemporânea.

\section{Palavras-chave}

Trabalho remoto. Teletrabalho. Produção científica. Covid-19. América Latina e Caribe.

\begin{abstract}
Home-office is a relevant topic in the working world and was impulsed by the Covid-19 pandemic, since it required an adaptation of work to the social distancing. This article analyzes the scientific output of Latin American and Caribbean countries on home-office in the two most recent decades. With a scientometric approach based on data from articles indexed in the Web of Science (WoS) and SCIELO Citation Index (SCiELO CI), it analyzes evolution of studies, regional and international journals, geographic and thematic distribution, and collaboration in papers co-authorship. The results show a developing theme, with intense growth since the Covid-19 pandemic, which combines a priority focus on local journals and languages with publication in international channels. The subject, which is more explored in Social Sciences, has low international collaboration, and is developed around central themes such as teleworking, health, education and Covid-19. It concludes that the impulse received by the subject during the Covid-19 pandemic tends to broaden and diversify the researches
\end{abstract}


and discussions on home-office as a temporary, exceptional or systematic form of work in contemporary society.

\section{Keywords}

Home-office. Teleworking. Scientific output. Covid-19. Latin America and Caribbean.

\section{INTRODUÇÃO}

A pandemia instaurada a partir de 2019 e que assola a sociedade é um reflexo da ameaça global do novo coronavírus, denominado SARS-CoV-2, causador da doença Covid-19 (LANA et al., 2020). Devido ao alto grau de transmissibilidade e propagação do vírus na população mundial, a maioria dos países adotou como medida preventiva o distanciamento social. Em função do avanço acelerado da Covid-19 em um curto período de tempo e das medidas restritivas impostas pelas autoridades governamentais de cada país no atual contexto pandêmico, o home-office, considerado um tipo de trabalho remoto, ganhou notória relevância (MENDES; HASTENREITER FILHO; TELLECHEA, 2020).

O home-office não foi criado a partir do surgimento da pandemia do novo coronavírus, pelo contrário, essa prática existe há várias décadas como uma modalidade de teletrabalho (ROSENFIELD; ALVES, 2011). O fato é que a adoção do home-office, tanto por parte de instituições públicas e privadas quanto por parte de trabalhadores autônomos, desde o início da crise humanitária que aflige o planeta, parece ter sido impulsionado não só pelo avanço das tecnologias da informação e da comunicação (TICs) e das mudanças comportamentais da sociedade (BARROS; SILVA, 2010), mas também pelo uso crescente de plataformas digitais e de dispositivos móveis tais como computadores, tablets e smartphones.

Diante do contexto social apresentado, questiona-se: Quais as características da produção científica sobre home-office da América Latina e Caribe (ALC) no período de 20002020? Para poder responder essa questão, a pesquisa tem como objetivo geral identificar e analisar o volume de artigos científicos publicados em periódicos indexados pelas bases de dados multidisciplinares Web of Science (WOS) e SciELO Citation Index (SciELO CI). Os objetivos específicos da pesquisa têm a finalidade de analisar a evolução temporal das publicações sobre home-office, os periódicos de circulação regional e internacional, a distribuição geográfica e temática e a colaboração através dos artigos em coautoria, entre outros aspectos. Por meio de indicadores cientométricos, os resultados podem favorecer a compreensão do desenvolvimento e abordagem do tema nos países da região e o possível impulso das pesquisas no contexto da pandemia de Covid-19. A modalidade de teletrabalho home-office e suas implicações tem se tornado um fenômeno social interessante para explorar, principalmente nos últimos anos com o avanço da pandemia do coronavírus e a necessidade do distanciamento social. Justifica-se a importância da pesquisa para a literatura sobre homeoffice, considerando que se trata de um assunto atual e de extrema relevância para o momento pandêmico pelo qual a sociedade está atravessando. Cabe destacar que a temática explorada está fortemente alinhada à área acadêmica.

A WoS e a SciELO Cl são fontes complementares para a análise da produção científica da ALC, bastante utilizadas em estudos métricos da informação. Recentemente, comprovouse um aumento significativo do número de publicações da ALC indexadas na WoS (SANTIN; CAREGNATO, 2020). Parte desse crescimento é decorrente das mudanças implementadas pela WoS na sua política editorial, ocasionando um incremento considerável do número de periódicos regionais (TESTA, 2011; COLLAZO-REYES, 2014; VÉLEZ-CUARTAS; LUCIO-ARIAS; 
LEYDESDORFF, 2016), e da criação do Emerging Sources Citation Index (ESCI), em 2015, com maior cobertura das revistas regionais. O uso complementar da WoS e SciELO Cl contribui para uma maior visibilidade e reconhecimento da ciência latino-americana e para a geração de indicadores mais adequados de produção científica da ALC.

\section{A MODALIDADE DE TELETRABALHO HOME-OFFICE}

O momento pandêmico pelo qual a sociedade está atravessando exigiu mudanças e novas configurações comportamentais (MENDES; HASTENREITER FILHO; TELLECHEA, 2020) em escala mundial. O home-office pode ser considerado uma estratégia apropriada e necessária, de caráter provisório (RAFALSKI; ANDRADE, 2015), excepcional ou sistemático que diversas empresas encontraram para desenvolver suas atividades econômicas desde meados de março de 2020, contribuindo para a diminuição da circulação e aglomeração de indivíduos e a desaceleração do contágio por coronavírus. A adaptação ao home-office no contexto da pandemia não é um processo simples, requer um esforço grande de todos os envolvidos para manter o nível de produtividade (RAFALSKI; ANDRADE, 2015) e qualidade do trabaIho a ser realizado. A retomada às atividades presenciais com segurança e sem risco para a população dependerá da eficácia dos programas de imunização implementados recentemente no combate à Covid-19.

A diminuição da taxa de transmissão e letalidade comunitária da doença Covid-19 tem sido uma preocupação constante das autoridades sanitárias desde o início da pandemia. Nesse sentido, a modalidade de trabalho home-office se apresentou como uma alternativa plausível perante a gravidade das circunstâncias, uma vez que as pessoas em confinamento têm a possibilidade de trabalhar isoladamente dentro do seu espaço privado de convívio, evitando o risco de contágio.

Embora o trabalho em casa ou home-office, realizado de forma descentralizada através de plataformas móveis e softwares corporativos, tenha começado a crescer em popularidade a partir dos anos 2000, há indícios da adoção dessa modalidade profissional desde a década de 1970 (RAFALSKI; ANDRADE, 2015). De modo mais abrangente, o teletrabalho pode ser definido como uma modalidade flexível de organização laboral onde os trabalhadores desempenham as atividades profissionais de forma remota, desde a sua casa, escritório virtual ou local alternativo, não necessariamente todos os dias da semana, durante ou fora do horário de expediente, utilizando diversos recursos tecnológicos de comunicação (LIM; TEO, 2000).

No contexto da pandemia causada pelo novo coronavírus, o teletrabalho assumiu transitoriamente a forma de home-office, caracterizado como o trabalho realizado em casa, seja em caráter provisório ou definitivo (RAFALSKI; ANDRADE, 2015). Existem diversas modalidades de exercer a atividade laboral no âmbito do teletrabalho, sendo o home-office, ou seja, o trabalho realizado na residência do trabalhador, a mais comum de todas (HAZAN; MORATO, 2018). O home-office é um regime no qual os indivíduos realizam a maior parte do trabalho fora do espaço físico da empresa à qual se encontram vinculados profissionalmente (BARROS; SILVA, 2010).

No âmbito nacional, por exemplo, a relevância do home-office como modalidade de teletrabalho instaurada no contexto pandêmico atual é posta de manifesto em diversos estudos estatísticos por indicadores sociais e econômicos. Com dados de 139 empresas, com destaque para os setores da indústria, comércio e serviços, que atuam em todo o Brasil, na pesquisa "Gestão de Pessoas na Crise da Covid-19" constatou-se que o home-office foi ado- 
tado por $46,0 \%$ da totalidade delas durante a pandemia (FUNDAÇÃO INSTITUTO DE ADMINISTRAÇÃO, 2020). Com vistas ao futuro, a pesquisa realizada por Cushman \& Wakefield aponta que $74,0 \%$ das empresas no Brasil pretendem implantar o home-office de forma definitiva no período pós-pandêmico (FLACH, 2020).

Apesar do otimismo das organizações quanto à viabilidade e sucesso na implantação dessa modalidade de trabalho na sociedade brasileira, o home-office, no entanto, parece ser um benefício do qual nem todos podem usufruir igualitariamente. A pesquisa PNAD Covid19 mostrou que apenas 7,9 milhões de trabalhadores brasileiros se encontravam em trabaIho remoto em 2020 (INSTITUTO BRASILEIRO DE GEOGRAFIA E ESTATÍSTICA, 2020), equivalente a $8,2 \%$ da população economicamente ativa $(96,1$ milhões de pessoas) conforme PNAD de 2020 elaborado pelo IBGE (MINISTÉRIO DA ECONOMIA, 2020). Certamente, o home-office pode acentuar as desigualdades sociais e evidenciar a informalidade laboral não apenas em nível nacional, mas também em nível regional, beneficiando pessoas com maior nível de instrução (superior completo ou pós-graduação), tal como se observa na pesquisa PNAD Covid19 (INSTITUTO BRASILEIRO DE GEOGRAFIA E ESTATÍ́STICA, 2020).

$\mathrm{Na}$ literatura sobre o tema podem ser identificadas diversas vantagens e desvantagens do home-office. A flexibilidade de horários, redução de custos, aumento da produtividade e qualidade de vida e do trabalho são apontados como aspectos favoráveis e motivadores para o investimento no home-office (BARROS; SILVA, 2010; RAFALSKI; ANDRADE, 2015; FILARDI, CASTRO; ZANINI, 2020). Entretanto, a falta de socialização, a dificuldade em estabelecer limites entre o ambiente pessoal e profissional e o problema para manter a concentração por muito tempo pode diminuir o grau de aceitação do trabalho remoto por parte dos trabalhadores (RAFALSKI; ANDRADE, 2015). A respeito das vantagens, entende-se que a redução de custos pode ter tido impacto positivo para empresas de diversos setores, mas não para os trabalhadores, uma vez que estes tiveram que arcar com os custos do home-office (energia elétrica, internet, equipamentos etc.).

A falta de socialização pode ser um aspecto diretamente relacionado a outros tipos de desvantagens decorrentes do home-office como, por exemplo, a dificuldade de comunicação, quase que restrita ao meio eletrônico e a menor visibilidade e contato dos indivíduos devido ao distanciamento físico (BARROS; SILVA, 2010). O isolamento social, problemas de infraestrutura tecnológica e falta de adaptação ao trabalho remoto são fatores que precisam ser analisados com cautela, já que podem causar sérios danos à saúde dos trabalhadores, tais como depressão e doenças psicológicas (FILARDI; CASTRO; ZANINI, 2020).

\section{METODOLOGIA}

O estudo cientométrico desenvolvido se enquadra dentro das pesquisas de nível macro, envolvendo a produção científica de uma região. A busca e coleta dos registros bibliográficos da ALC na temática home-office foi realizada em 14 de maio de 2021, através da busca avançada, nos índices da WoS - Science Citation Index Expanded (SCl), Social Sciences Citation Index (SSCl), Arts \& Humanities Citation Index (A\&HCl) e Emerging Sources Citation Index (ESCl) e no SciELO Citation Index (SciELO Cl). A pesquisa inclui os artigos, originais e de revisão, uma vez que são considerados os tipos de documentos mais utilizados na comunicação científica, o que acaba refletindo na visibilidade e impacto que adquirem desde o momento que são publicados.

O corpus da pesquisa é constituído por 79 artigos da WoS e 46 artigos do SciELO Cl, totalizando 125 artigos. A combinação de dados das duas fontes promove a composição de 
um corpus abrangente em relação à cobertura dos artigos latino-americanos de circulação regional e internacional. A limpeza manual de dados assegurou a exclusão de artigos que não tratavam do teletrabalho na modalidade home-office como tema de pesquisa, além de artigos duplicados. Ressalta-se que, a pesquisa tem como principal objeto de estudo o homeoffice, uma vez que o trabalho em casa é a modalidade de teletrabalho mais popular. Apenas dois artigos constavam nas duas bases de dados, e tiveram os dados da SciELO excluídos, a fim de evitar a duplicidade. A delimitação temporal de 20 anos visa analisar a evolução temporal das publicações sobre home-office desde a época em que começou a tornar-se popular como modalidade de teletrabalho até a atualidade no contexto de pandemia.

Na recuperação das publicações, combinaram-se os campos: Country (CU), Topic (TS), Document Type (DT), e Year Published (PY). Em concordância com a estratégia utilizada na investigação de Santin e Caregnato (2020), os nomes dos 33 países e 17 territórios dependentes da ALC foram coletados na versão on-line da publicação Standard Country or Area Codes for Statistics Use (UNITED NATIONS, 2021). Com o intuito de potencializar a busca dos registros bibliográficos na WoS, foram incluídas variantes dos nomes dos países nos idiomas espanhol e português. Com uso de operadores booleanos, a estratégia de busca foi definida da seguinte maneira para as duas bases de dados, com adaptação do campo DT (researcharticle OR review-article) na SciELO Cl, conforme apresentado no Quadro 1.

Quadro 1 - Estratégia de busca para constituição do corpus de pesquisa

\begin{tabular}{|c|}
\hline ome-nf \\
\hline $\begin{array}{l}\text { CU=(Anguilla OR "Antigua y Barbuda" OR "Antigua \& Barbuda" OR "Antígua e Barbuda" OR Aruba } \\
\text { OR Bahamas OR Baamas OR Barbados OR Bermuda OR Bonaire OR "Bonaire, Sint Eustatius and } \\
\text { Saba" OR "British Virgin Islands" OR "Islas Vírgenes" OR "Virgin Islands" OR "Ilhas Virgens" OR "Islas } \\
\text { Caimán" OR "Cayman Islands" OR "Ilhas Caimão" OR Cuba OR Curaçao OR Dominica OR Domínica } \\
\text { OR "República Dominicana" OR "Dominican Republic" OR Grenada OR Granada OR Guadalupe OR } \\
\text { Guadeloupe OR Haití OR Haiti OR Jamaica OR Martinica OR Martinique OR Montserrat OR "Puerto } \\
\text { Rico" OR "Porto Rico" OR "San Bartolomé" OR "Saint Barthélemy" OR "San Cristóbal y Nieves" OR } \\
\text { "St. Kitts \& Nevis" OR "São Cristóvão e Neves" OR "Saint Kitts and Nevis" OR "Saint Kitts \& Nevis" } \\
\text { OR "Saint Lucia" OR "Santa Lucía" OR "St. Lucia" OR "Santa Lúcia" OR "Saint Martin" OR "Sint } \\
\text { Maarten" OR "San Vicente y las Granadinas" OR "St. Vicent and the Grenadines" OR "São Vicente e } \\
\text { Granadinas" OR "Trinidad y Tobago" OR "Trinidad \& Tobago" OR "Trinidade Tobago" OR "Islas Turcas } \\
\text { y Caicos" OR "Turks \& Caicos Islands" OR "Ilhas Turcas e Caicos" OR "United States Virgin Islands" } \\
\text { OR Belice OR Belize OR "Costa Rica" OR "EI Salvador" OR Guatemala OR Honduras OR Mexico OR } \\
\text { México OR Méjico OR Nicaragua OR Nicarágua OR Panama OR Panamá OR Argentina OR Bolivia OR } \\
\text { Bolívia OR Brasil OR Brazil OR "Bouvet Island" OR Chile OR Colombia OR Colômbia OR Ecuador OR } \\
\text { Equador OR "Falkland Islands" OR "Islas Malvinas" OR "French Guiana" OR "Guyana Francesa" OR } \\
\text { "Guiana Francesa" OR Guiana OR Guyana OR Paraguay OR Paraguai OR Peru OR Perú OR "South } \\
\text { Georgia and the South Sandwich Islands" OR Suriname OR Surinam OR Uruguai OR Uruguay OR } \\
\text { Venezuela) AND TS=("home office" OR "home-office" OR teletrabalho OR teletrabajo OR telework } \\
\text { OR teleworking OR "trabalho remoto" OR "remote work" OR "trabajo remoto" OR "work at home" } \\
\text { OR "trabalho em casa" OR "trabajo en casa" OR "work from home" OR "trabalho a partir de casa" } \\
\text { OR "trabajo desde casa" OR "home-based work" OR "trabalho domiciliar") AND DT=(article OR } \\
\text { review) AND PY=(2000-2020). }\end{array}$ \\
\hline
\end{tabular}

Fonte: elaborado pelos autores (2022).

Os dados foram importados da WoS e da SciELO Cl em formatos de arquivos de texto (.txt) e reunidos em arquivo único para as duas bases de dados com auxílio do software Bi- 
bexcel $^{1}$. Os nomes dos países mais produtivos foram manualmente padronizados para a geração da frequência. Os dados foram analisados por meio de indicadores cientométricos de produção científica (ano de publicação, periódico, áreas de pesquisa, países, coocorrência de palavras) e colaboração (em nível de países). Enquanto o Bibexcel possibilitou a leitura dos dados bibliográficos e geração das frequências, o Microsoft Excel auxiliou na elaboração das tabelas e figuras apresentadas e o VOSviewer ${ }^{2}$ foi utilizado para os mapas temáticos e redes de colaboração. Esta pesquisa utilizou a contagem total (GLÄNZEL, 2003), ou seja, com a atribuição de um artigo ou citação para cada uma das áreas e dos países dos autores que assinaram a publicação.

\section{RESULTADOS E DISCUSSÃO}

A produção científica da ALC na temática home-office de 2000-2020 indexada na WoS e SciELO Cl é constituída por 125 publicações: 119 artigos originais (95,2\%) e 6 artigos de revisão (4,8\%). É justificável a representatividade do artigo original como tipo de documento, uma vez que é o principal veículo de publicação no processo de comunicação científica (GOLDSCHMIDT-CLERMONT, 2002). Além disso, os artigos são o principal tipo de documento nas duas fontes e a coleta de dados da pesquisa delimitou os resultados a esse tipo de publicação. Os artigos em espanhol representam 39,2\%, enquanto o inglês ocorre em 33,6\%, o português, em $26,4 \%$ e o francês, em $0,8 \%$. Os artigos em inglês representam $53,0 \%$ na WoS, ou seja, pouco mais da metade do total da produção. A prevalência do inglês era presumível na base internacional, uma vez que esta prioriza a indexação de revistas na língua inglesa. Entretanto, é necessário destacar o protagonismo de publicações sobre home-office em idiomas locais, espanhol e português. As publicações em ambos os idiomas representam $65,6 \%$ das pesquisas indexadas nas duas bases, evidenciando a relevância dos idiomas locais nessa temática, que se desenvolve especialmente nas Ciências Sociais.

A distribuição anual das publicações revela um baixo volume da produção científica no período de 2000-2014. As primeiras pesquisas analisaram a viabilidade dos novos modelos organizacionais de trabalho, de caráter mais flexível, no contexto evolutivo dos serviços de telecomunicações. Nesse cenário, estudo desenvolvido em uma empresa de processamento de dados concluiu que o trabalho em casa seria factível, desde que fossem asseguradas às pessoas as mesmas condições organizacionais, técnicas, psicológicas e legais (FETZNER, 2001). A partir de 2015 há maior impulso nas pesquisas, com 10 artigos publicados, e as investigações começam a ser desenvolvidas com maior intensidade e sob diversas perspectivas.

Pesquisas comprovaram que indivíduos que têm a oportunidade de trabalhar em casa são mais engajados e eficazes do que aqueles que não têm essa chance (MASUDA; HOLTSCHLAG; NICKLIN, 2017). Apesar dos potenciais benefícios que o trabalho domiciliar pressupõe oferecer tanto para os funcionários quanto para as organizações, em países da ALC como Brasil, foi evidenciado que ainda não existe uma regulamentação consolidada acerca do teletrabalho (incluindo o home-office), a tal ponto de garantir aos teletrabalhadores todos seus direitos trabalhistas (HAZAN; MORATO, 2018).

O crescimento da produção sobre home-office é nitidamente perceptível em 2020 (Gráfico 1), coincidindo com o ano de início da pandemia causada pelo novo coronavírus. As publicações de 2020 representam quase $40,0 \%$ dos artigos. No contexto pandêmico atual, a

\footnotetext{
${ }^{1}$ Disponível em: https://homepage.univie.ac.at/juan.gorraiz/bibexcel/

${ }^{2}$ Disponível em: https://www.vosviewer.com/
} 
modalidade home-office foi percebida como uma possibilidade concreta de trabalho devido ao alto risco de transmissão da Covid-19 por contato pessoal, trazendo vários desafios e mudanças na organização do ambiente laboral dos trabalhadores. Tal situação impulsionou as pesquisas na temática e os debates sobre as implicações sociais dessa prática.

Em tempos de pandemia, as investigações tiveram como uma das temáticas prioritárias para análise o impacto do teletrabalho na modalidade home-office na saúde dos trabaIhadores. Nesse sentido, observou-se que os efeitos negativos que particularmente podem comprometer a saúde ocupacional das pessoas em trabalho remoto estão relacionados, dentre outros fatores, com a fadiga e a carga mental (VENEGAS TRESIERRA; LEYVA POZO, 2020), com o confinamento domiciliar (VOLINO-SOUZA et al., 2020), com os conflitos trabaIho-família, influenciados pela falta de um ambiente apropriado, presença de familiares enquanto se trabalha e sobrecarga de trabalho (SOLís, 2016) e com a falta de infraestrutura tecnológica. No cenário atual, o home-office pode ser bem-sucedido desde que seja possível reverter a situação de estresse e angústia provocada pela Covid-19 através de ações que visem a melhoria das condições laborais (GÓMEZ et al., 2020) e a ergonomia dos escritórios domésticos (RALPH et al., 2020). Em pesquisa recente, algumas das entrevistadas fazem questão de enfatizar a sobrecarga de trabalho, entretanto, destacam como efeitos positivos do home-office a proximidade com a família decorrente da nova rotina e a maior disponibilidade de tempo para atividades físicas e de lazer (LEMOS; BARBOSA; MONZATO, 2020).

Gráfico 1 - Evolução da produção latino-americana sobre home-office (2000-2020)

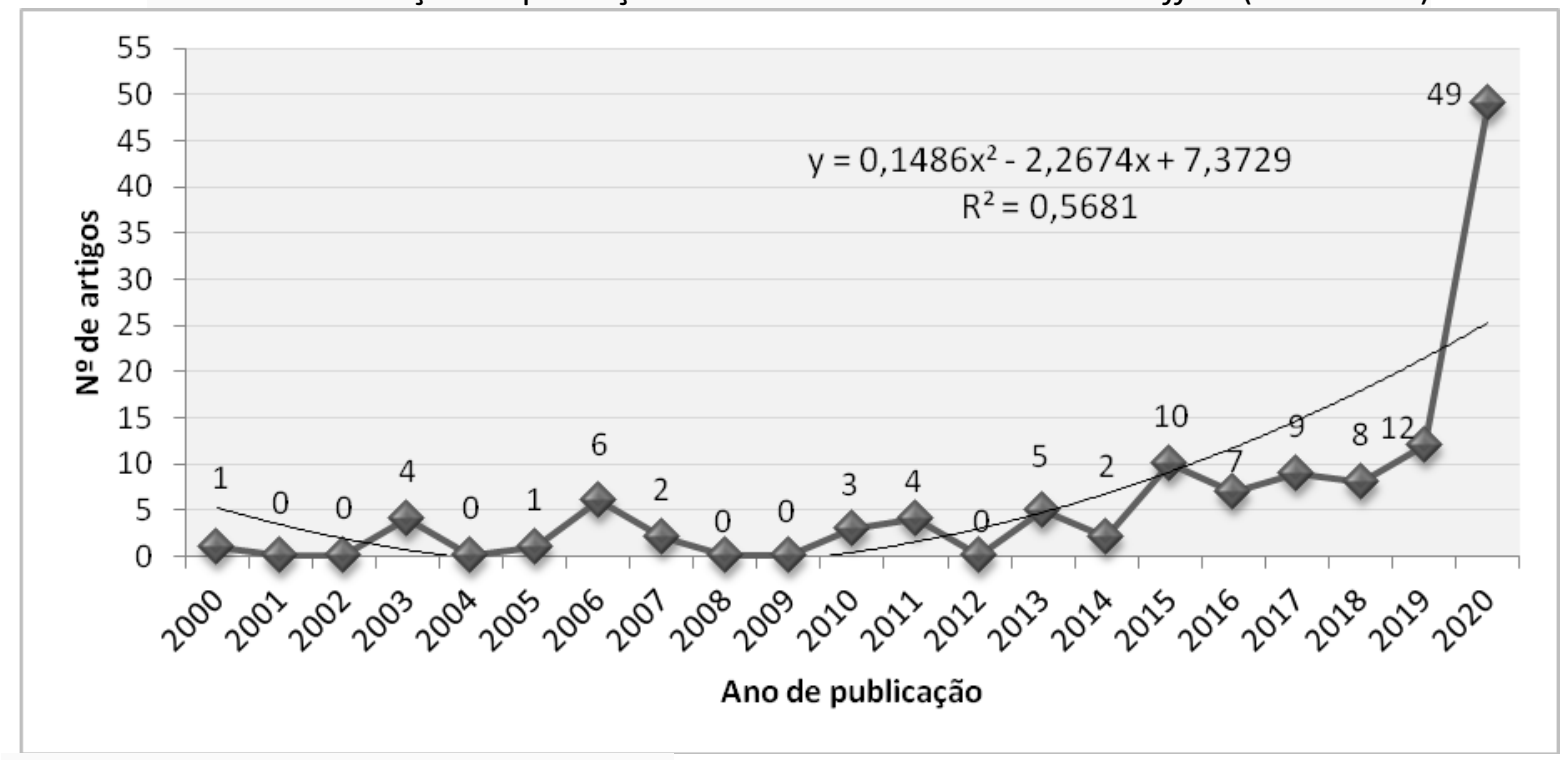

Fonte: elaborado pelos autores (2021).

A distribuição dos 125 artigos da ALC na temática home-office de 2000-2020 por periódico mostra uma ampla dispersão da produção, uma vez que foram utilizados 108 veículos na comunicação científica, sendo $76(70,4 \%)$ indexados na WoS e $32(29,6 \%)$ na SciELO Cl. $O$ indicador evidencia a ampla gama de revistas e abordagens de pesquisa que podem ser utilizadas pelas diferentes áreas para explorar um mesmo assunto. Nos periódicos identificados, o teletrabalho na forma de home-office foi analisado pela comunidade científica da ALC sob diferentes perspectivas, a fim de contemplar aspectos inerentes a essa modalidade profissional relacionado com direitos trabalhistas (legislação e regulamentação), tecnologia da informação e da comunicação (ferramentas tecnológicas de telecomunicação), adminis- 
tração (gestão de pessoas, habilidades e competências comportamentais, produtividade) e saúde ocupacional (bem-estar dos indivíduos em trabalho remoto).

Os 65 periódicos editados por países da ALC representam 60,2\% dos títulos, sendo responsáveis pela publicação de 77 artigos (61,6\%), ou seja, a maior parte das publicações sobre a temática home-office. Embora predomine o espanhol e português em artigos científicos publicados nos periódicos locais, realçando uma característica importante da ciência regional (SANTIN; CAREGNATO, 2020), observam-se alguns periódicos publicados em inglês e francês. Os títulos da ALC estão concentrados em apenas oito países, com destaque para o Brasil com 28 (25,9\%) dos 65 periódicos editados na região. Além do Brasil, salienta-se a presença latino-americana e caribenha de periódicos editados na Colômbia (16), México (6), Cuba (5), Chile (4), Venezuela (4), Costa Rica (1) e Equador (1). O indicador revela a diversidade de publicações da região e o potencial de visibilidade dos periódicos locais indexados em bases regionais como a SciELO e internacionais, podendo contribuir para a difusão, visibilidade e impacto das publicações regionais sobre home-office da ALC.

Os periódicos editados por países fora da região representam 39,8\% do total de veículos utilizados na divulgação das investigações sobre home-office da ALC, sendo responsáveis por 48 trabalhos (38,4\%). Embora prevaleça o inglês nos artigos dos periódicos estrangeiros, há também publicações em espanhol e português. Os títulos estão vinculados a 10 países, com destaque para EUA e Inglaterra, uma vez que ambos detêm, em conjunto, 22 dos 43 periódicos editados fora da ALC. Na WoS, tanto o número de periódicos editados quanto de artigos é maior no conjunto de países não regionais. A soma do conjunto de dados da WoS e da SciELO Cl inverte a situação, com maior volume de periódicos e artigos publicados na região, o que reforça a importância da complementaridade das bases regionais e internacionais na avaliação da ciência e da produção científica.

A partir dos resultados obtidos, é possível aferir uma moderada internacionalização da produção científica sobre home-office da ALC, levando em consideração o número de artigos publicados em periódicos editados fora da região e o uso do idioma inglês. Esse comportamento pode justificar, ainda que, parcialmente, a pouca relação existente entre produtividade e impacto das publicações latino-americanas e caribenhas sobre home-office por periódico (Tabela 1), principalmente se for analisado o volume de citações recebidas pelos periódicos regionais. No período avaliado, verificou-se que os artigos da ALC receberam 215 citações, sendo que 167 (77,7\%) do total são oriundas de periódicos indexados na WoS. Por outro lado, os periódicos regionais publicados na $\mathrm{ALC}$ e indexados na SciELO $\mathrm{Cl}$, receberam somente 48 citações, ou seja, $22,3 \%$ do total.

Apesar da boa visibilidade que propicia a indexação paulatina de periódicos regionais em bases de dados internacionais como WoS, de modo geral há uma preocupação latente no que diz respeito ao desproporcional crescimento que se observa entre a produção científica e o impacto das publicações da ALC (SANTIN; CAREGNATO, 2020). Em particular, o baixo número de citações recebidas pelos artigos em espanhol e português sobre home-office pode ser um sinal do papel secundário que periódicos editados regionalmente exercem nas bases internacionais. É preciso considerar, porém, que boa parte dos artigos foi publicada em anos recentes, e que pode ser necessário mais tempo para o uso pela comunidade científica. Por fim, os países periféricos costumam dar mais citações das que recebem (RÀFOLS et al., 2016), inclusive no contexto da ALC, trazendo à tona e reforçando o lugar de privilégio que países líderes ocupam na ciência mundial (SANTIN; CAREGNATO, 2020). 
Tabela 1 - Periódicos de publicação dos artigos latino-americanos sobre home-office (2000-2020)

\begin{tabular}{llccccc}
\hline \multicolumn{1}{c}{ Periódico } & País & Artigos & $\%$ & Citações & $\%$ & $\begin{array}{c}\text { Média de } \\
\text { citações }\end{array}$ \\
\hline Cadernos EBAPE.BR & Brasil & 5 & 4,0 & 10 & 4,7 & 2,0 \\
Cyberpsychology \& Behavior & EUA & 4 & 3,2 & 7 & 3,3 & 1,7 \\
Ciencia \& Trabajo & Chile & 3 & 2,4 & 4 & 1,9 & 1,3 \\
Journal of Cleaner Production & Inglaterra & 2 & 1,6 & 30 & 14,0 & 15,0 \\
Dados - Revista de Ciências Sociais & Brasil & 2 & 1,6 & 13 & 6,0 & 6,5 \\
Organizações \& Sociedade & Brasil & 2 & 1,6 & 3 & 1,4 & 1,5 \\
Revista Ciências Administrativas & Brasil & 2 & 1,6 & 0 & 0,0 & 0,0 \\
Revista Conrado & Cuba & 2 & 1,6 & 0 & 0,0 & 0,0 \\
Revista General del Derecho del & Espanha & 2 & 1,6 & 0 & 0,0 & 0,0 \\
Trabajo y de la Seguridad Social & México & 2 & 1,6 & 0 & 0,0 & 0,0 \\
Revista IUS & -- & 59 & 47,2 & 42 & 19,5 & 0,7 \\
Outras revistas da ALC & -- & 40 & 32,0 & 106 & 49,3 & 2,6 \\
Outras revistas internacionais & & 125 & 100 & 215 & 100 & 1,7 \\
Total & & & & & & \\
\hline \multicolumn{1}{r}{ Fonte: elaborada pelos autores (2021). } & & & &
\end{tabular}

A distribuição geográfica dos artigos e citações latino-americanos sobre home-office (Tabela 2) destaca a liderança de Brasil, Colômbia e México e reforça a concentração das pesquisas em 11 dos 50 países e territórios que compõem a ALC. O Brasil lidera a produção científica sobre o tema, com pouco mais que o dobro das publicações que a Colômbia, que ocupa a segunda posição na produção e inverte a posição com México nas citações. Com pouco mais de $30 \%$ da população e $60 \%$ dos recursos humanos em ciência e tecnologia, o Brasil reúne mais de $50 \%$ dos artículos da região, além de ser o país que mais investe em ciência (SANTIN; CAREGNATO, 2020; RICYT, 2020). No tema home-office, o Brasil reúne $44,0 \%$ dos artigos e $58,1 \%$ das citações, alcançando a terceira maior média de citações da região ( 2,3$)$, antecedido pela Jamaica (3,0 citações/artigo) e México (2,6 citações/artigo).

Tabela 2 - Artigos e citações sobre home-office por país da ALC (2000-2020)

\begin{tabular}{lccccc}
\hline \multicolumn{1}{c}{ País } & Artigos & \% & Citações & \% & Média de citações \\
\hline Brasil & 55 & 44,0 & 125 & 58,1 & 2,3 \\
Colômbia & 27 & 21,6 & 22 & 10,2 & 0,8 \\
México & 14 & 11,2 & 37 & 17,2 & 2,6 \\
Chile & 7 & 5,6 & 11 & 5,1 & 1,6 \\
Venezuela & 6 & 4,8 & 2 & 0,9 & 0,3 \\
Peru & 5 & 4,0 & 5 & 2,3 & 1,0 \\
Argentina & 5 & 4,0 & 4 & 1,9 & 0,8 \\
Costa Rica & 5 & 4,0 & 6 & 2,8 & 1,2 \\
Ecuador & 3 & 2,4 & 2 & 0,9 & 0,7 \\
Cuba & 3 & 2,4 & 2 & 0,9 & 0,7 \\
Jamaica & 1 & 0,8 & 3 & 1,4 & 3,0 \\
\hline
\end{tabular}

Fonte: elaborada pelos autores (2021). 
Ocorre uma inversão nas posições dos países quanto ao número de artigos publicados frente à produção geral da ALC no início do século XXI (SANTIN; CAREGNATO, 2020). Na produção geral, as primeiras posições são ocupadas por Brasil, México, Argentina, Chile, Colombia e Venezuela, nesta ordem. Nos artigos sobre home-office, Colômbia assume maior destaque e vários países precedem a Argentina, por exemplo. Em relação às sub-regiões, a América do Sul mantém o destaque regional com os países mais produtivos, a América Central está representada pela Costa Rica e o Caribe, por Cuba e Jamaica. Em relação ao impacto de citações, a média de citações dos países da ALC no tema home-office é menor que a média da produção geral da ciência regional, inclusive das Ciências Sociais. Os artigos regionais de várias áreas indexados no SciELO Cl têm em média 1,9 citações, enquanto na WoS esse valor eleva-se para 12,8 citações por artigo (SANTIN; CAREGNATO, 2020).

Home-office é um tema de pesquisa multidisciplinar e sua produção acadêmica envolve contribuições de diferentes áreas e disciplinas. A Tabela 3 apresenta a distribuição dos artigos com base na classificação das áreas de pesquisa da WoS e SciELO (campo Research Areas - SC) no nível meso, e nos seis campos principais de ciência e tecnologia (C\&T) do Manual Frascati (OECD, 2007), no nível macro. Observa-se que home-office constitui especialmente um tema de pesquisa das Ciências Sociais $(76,8 \%)$, Engenharia \& Tecnologia $(19,2)$ e Ciências Médicas \& da Saúde (15,2\%). As Ciências Sociais têm maior tradição nas pesquisas e nos debates sobre o trabalho e maior produção nas duas décadas na temática, principalmente nas áreas de Economia \& Negócios (24,8\%), Ciências Sociais (8,8\%), Governo \& Direito $(8,0 \%)$, Educação \& Pesquisa Educacional (7,2\%) e Psicologia (7,2\%), Administração Pública (4\%) e Sociologia (4,0\%), além de outras disciplinas. Engenharia \& Tecnologia têm importantes contribuições no assunto, com enfoque no trabalho e tecnologias, enquanto as Ciências Médicas \& da Saúde abordam aspectos da saúde e qualidade de vida, com destaque para Saúde Pública, Ambiental e Ocupacional (8,8\%).

Tabela 3 - Distribuição dos artigos da ALC sobre home-office por área e campo de C\&T (2000-2020)

\begin{tabular}{|c|c|c|c|c|}
\hline Campo de C\&T & $\%$ & Área de Pesquisa & Artigos & $\%$ \\
\hline \multirow{15}{*}{ Ciências Sociais } & \multirow{15}{*}{76,8} & Economia \& Negócios & 31 & 24,8 \\
\hline & & Ciências Sociais - Outros Tópicos & 11 & 8,8 \\
\hline & & Governo \& Direito & 10 & 8,0 \\
\hline & & Educação \& Pesquisa Educacional & 9 & 7,2 \\
\hline & & Psicologia & 9 & 7,2 \\
\hline & & Administração Pública & 5 & 4,0 \\
\hline & & Sociologia & 5 & 4,0 \\
\hline & & Comunicação & 4 & 3,2 \\
\hline & & Geografia & 3 & 2,4 \\
\hline & & Ciência da Informação \& Biblioteconomia & 2 & 1,6 \\
\hline & & Questões Sociais & 2 & 1,6 \\
\hline & & Estudos Urbanos & 2 & 1,6 \\
\hline & & Demografia & 1 & 0,8 \\
\hline & & Estudos da Família & 1 & 0,8 \\
\hline & & Serviço Social & 1 & 0,8 \\
\hline & \multirow[b]{4}{*}{19,2} & Engenharia & 8 & 6,4 \\
\hline & & Ciência da Computação & 7 & 5,6 \\
\hline & & Ciência e Tecnologia - Outros Tópicos & 5 & 4,0 \\
\hline Engenharia \& Tecnologia & & Telecomunicações & 2 & 1,6 \\
\hline
\end{tabular}




\begin{tabular}{|c|c|c|c|c|}
\hline & & Energia \& Combustíveis & 1 & 0,8 \\
\hline & & Transporte & 1 & 0,8 \\
\hline \multirow{6}{*}{ Ciências Médicas \& da Saúde } & \multirow{6}{*}{15,2} & Saúde Pública, Ambiental \& Ocupacional & 11 & 8,8 \\
\hline & & Medicina Geral \& Interna & 2 & 1,6 \\
\hline & & Gastroenterologia \& Hepatologia & 1 & 0,8 \\
\hline & & Doenças Infecciosas & 1 & 0,8 \\
\hline & & Enfermagem & 1 & 0,8 \\
\hline & & Nutrição \& Dietética & 1 & 0,8 \\
\hline \multirow{3}{*}{ Humanidades } & \multirow{3}{*}{3,2} & Artes \& Humanidades - Outros Tópicos & 2 & 1,6 \\
\hline & & Arquitetura & 1 & 0,8 \\
\hline & & Religião & 1 & 0,8 \\
\hline & \multirow[t]{2}{*}{4,0} & Ciências Ambientais \& Ecologia & 3 & 2,4 \\
\hline Ciências Naturais & & Geografia Física & 2 & 1,6 \\
\hline Ciências Agrárias & 0,8 & Agricultura & 1 & 0,8 \\
\hline
\end{tabular}

Fonte: elaborada pelos autores (2021).

Nota: o mesmo artigo pode ser classificado em mais de uma área, de modo que o total da contagem pode não coincidir com o total de artigos.

A Figura 1 apresenta o mapa de coocorrência de palavras-chave dos autores (campo Author Keywords $-D E$ ) que revela os agrupamentos temáticos na produção científica latinoamericana sobre home-office. Foram consideradas as palavras-chave que alcançaram o critério mínimo de duas ocorrências, o que resulta em 59 termos. Os clusteres foram definidos pelo método de associação do software VOSviewer e com o critério mínimo de 10 palavraschave para cada, de modo a permitir o olhar sobre o agrupamento de termos na temática.

Figura 1 - Coocorrência de palavras nos artigos latino-americanos sobre home-office (2000-2020)

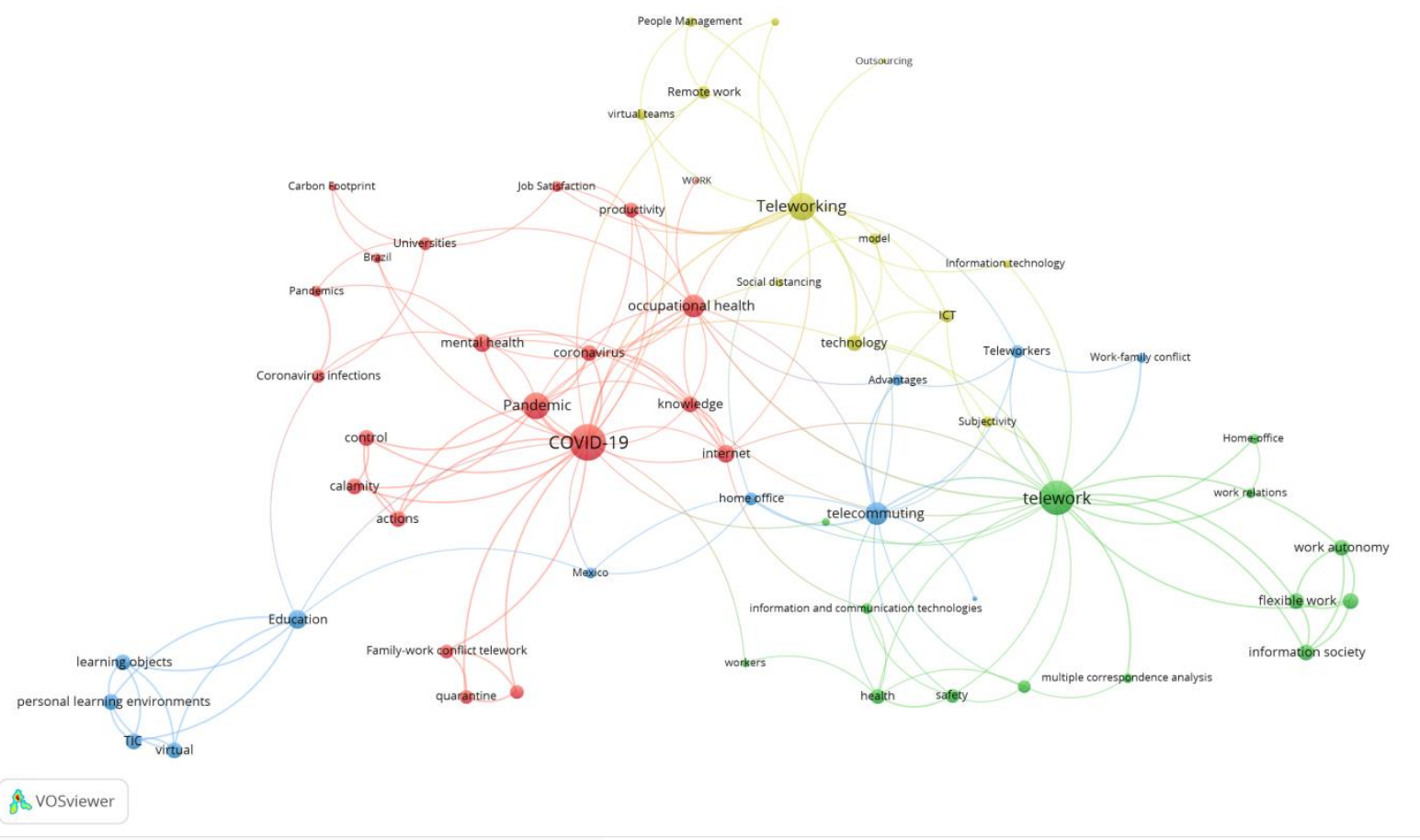

Fonte: elaborada pelos autores (2021). 
Os quatro clusters temáticos nos estudos latino-americanos sobre home-office são: a) em vermelho, o maior cluster, com 21 termos relacionados à pandemia Covid-19, que exigiu o distanciamento social e a adaptação para o trabalho remoto, reunindo termos como pandemia, coronavírus, saúde mental e ocupacional, conhecimento, produtividade, satisfação no trabalho, universidades, quarentena, conflitos família-trabalho, entre outros; b) em verde, o cluster do teletrabalho, com 14 termos, como home-office, trabalho flexível, saúde, segurança, tecnologias de informação e comunicação, trabalhadores, entre outros; c) em azul, com 12 termos, temas como telecomunicações, teletrabalhadores, educação, objetos de aprendizagem, virtual e outros; d) em amarelo, também com 12 termos, o cluster do teletrabalho, agrupando termos como trabalho remoto, tecnologias, equipes virtuais, terceirização, subjetividade e distanciamento social.

Observa-se um entrecruzamento dos temas em diferentes clusteres, indicando a diferentes enfoques de um tema multidisciplinar e em desenvolvimento. A pesquisa científica é altamente dinâmica e os focos temáticos podem mudar de maneira drástica, uma vez que as áreas e disciplinas se transformam e novos assuntos emergem espontaneamente no decorrer do tempo (MANE; BORNER, 2004).

Outro aspecto importante na produção de conhecimento acadêmico é a colaboração. Nos estudos latino-americanos sobre home-office, o índice de coautoria, representado pelo número médio de autores por artigo, foi de 2,8 , e a mediana de autores ficou em 2,0. Outro indicador importante é o grau de colaboração, representado pela proporção de trabalhos com mais de um autor, que ficou em $75,2 \%$, ou seja, 94 artigos em coautoria. Por outro lado, 31 artigos foram publicados com autoria única $(24,8 \%)$, um valor relativamente elevado, mas adequado aos padrões das Ciências Sociais, que em geral registram menor número de autores (OLIVEIRA, 2018; ALVAREZ; CAREGNATO, 2021) que as chamadas ciências duras, por exemplo, cujos padrões de autoria envolvem, em geral, maior volume de autores por publicação (VANZ, 2009; ALVAREZ; VANZ; BARBOSA, 2017).

Os artigos sobre home-office registram baixa colaboração internacional, inclusive na comparação com a produção geral dos países na região (UNITED NATIONS EDUCATIONAL, SCIENTIFIC AND CULTURAL ORGANIZATION, 2015). Com 22 artigos em colaboração internacional $(17,6 \%)$ e maior proporção na WoS do que na SciELO Cl, observa-se baixa internacionalização no aspecto da colaboração internacional, assim como na publicação em revistas internacionais e em idioma inglês.

A coautoria com outros países tende a gerar maior visibilidade e impacto de citações (ROYAL SOCIETY, 2011; LOW, 2014), e a baixa colaboração pode ter implicações sobre esses aspectos. É preciso considerar que diversos temas e objetos das Ciências Sociais, como o home-office, podem ter abordagem e enfoque local, específicos ou mais vinculados ao entorno em se que produzem (ALIAGA, 2020), o que não reduz a importância das pesquisas e da produção científica nessas temáticas. A Figura 2 apresenta a rede de colaboração nos artigos sobre home-office da ALC. 
Figura 2 - Rede de colaboração internacional nos artigos sobre home-office (2000-2020)

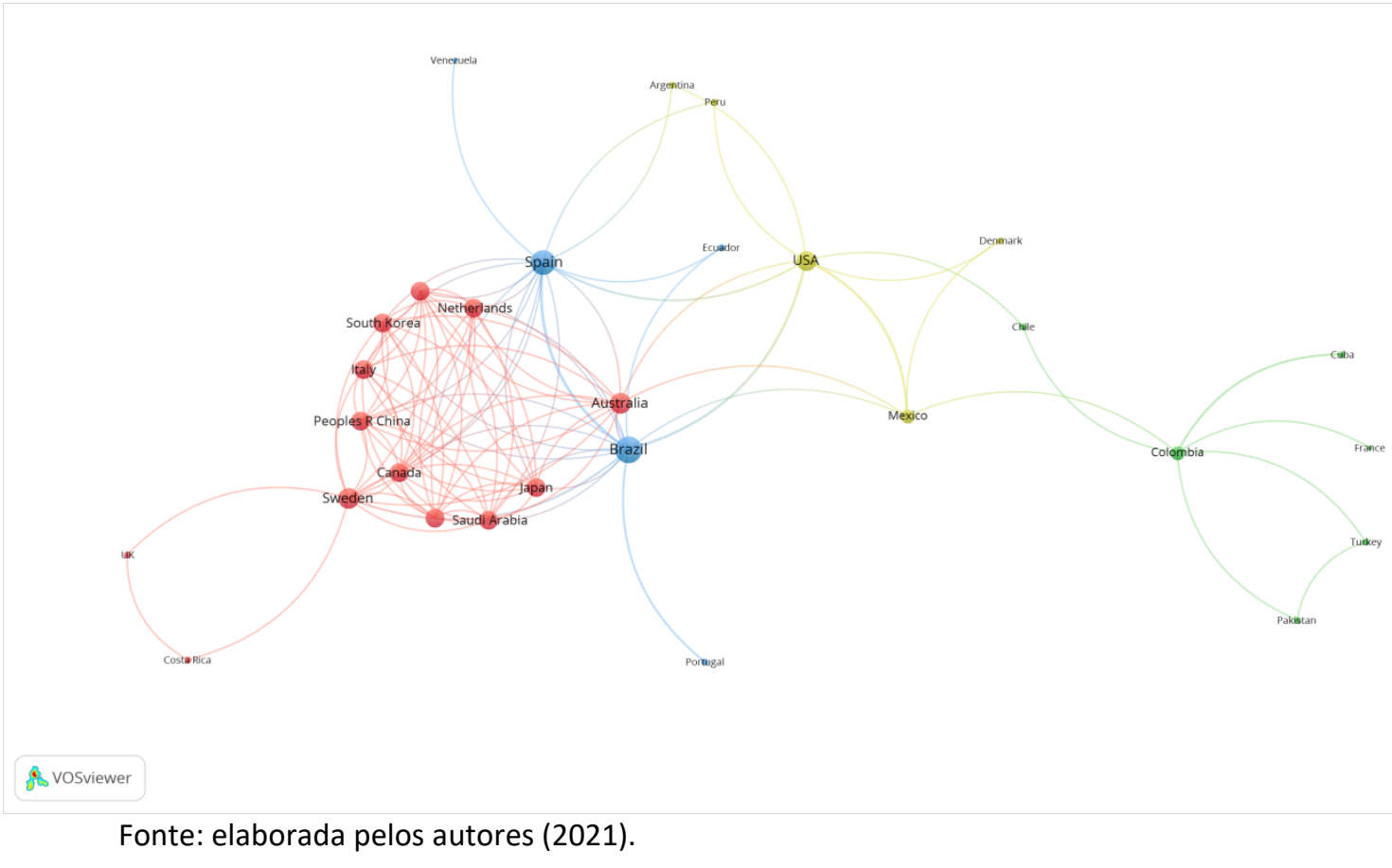

Os países mais produtivos da região nos estudos sobre home-office, Brasil, Colômbia e México são também os mais colaborativos, embora estabeleçam relações com outros países mais do que entre si. Há baixa colaboração intrarregional entre os países da ALC, com maior cooperação entre os países de idioma espanhol, apesar da liderança do Brasil em termos de produtividade e citações. A proximidade geográfica pode encorajar a colaboração (KATZ, 1994), entretanto, ela nem sempre é facilmente alcançada, já que diferentes padrões de trabalho, expectativas, crenças e objetivos pessoais podem dificultar a interação entre parceiros (HARA et al., 2003). Na colaboração internacional com países externos à ALC, os principais parceiros são Estados Unidos, Espanha e outros países europeus. Devido ao baixo volume de artigos, um grupo de países europeus, em vermelho na rede de colaboração internacional, assume destaque pela coautoria em poucos artigos, devendo ser observado com esta ressalva.

\section{CONSIDERAÇÕES FINAIS}

Em estudos cientométricos, a integração de bases de dados internacionais como a Web of Science e bases de dados regionais como a SciELO é particularmente relevante para captar desde diversos ângulos a realidade da investigação científica da ALC, em especial quando se trata de uma temática em desenvolvimento e inserida no atual contexto pandêmico, como é o caso do teletrabalho na modalidade home-office. O problema de pesquisa foi respondido e os objetivos devidamente alcançados, no entanto, é necessário destacar que, os indicadores cientométricos apresentados devem ser considerados como indicadores parciais, dado que expressam apenas as características da produção científica da ALC indexada em duas bases de dados. A produção científica da ALC sobre home-office no período 20002020 aumentou consideravelmente no último ano, como consequência da crise humanitária provocada pela Covid-19, que exigiu uma súbita adaptação do trabalho presencial para o remoto em favor da segurança sanitária e do distanciamento social. 
As implicações sociais decorrentes do home-office no âmbito do teletrabalho, saúde, tecnologia da informação e comunicação e educação impulsionaram as investigações com abordagem multidisciplinar, envolvendo contribuições de diferentes áreas e disciplinas, com destaque para as Ciências Sociais. É claramente apreciável a preferência dos pesquisadores latino-americanos para veicularem as descobertas científicas em periódicos e idiomas locais, realçando uma característica importante da ciência regional. Entretanto, é possível identificar também canais de comunicação internacionais nas publicações da ALC sobre homeoffice.

$\mathrm{Na}$ ALC, o Brasil assume isoladamente a liderança na produção científica sobre homeoffice, evidenciando o esforço dispensado em ciência e tecnologia nos últimos anos, mesmo com os sucessivos e crescentes cortes orçamentários nas universidades federais e centros de pesquisa da rede pública. A baixa colaboração internacional identificada nos artigos da ALC sobre home-office pode simbolizar parcialmente preocupações e interesses locais de pesquisa reais no contexto pandêmico da Covid-19. A baixa internacionalização nas publicações pode ser motivo para discutir a reformulação de políticas científicas e a distribuição de recursos nacionais que visem uma maior integração da ciência regional na produção científica mundial. Por fim, espera-se que os indicadores apresentados possam estimular, principalmente na ALC, a ampliação e diversificação das pesquisas e discussões sobre o home-office como forma de trabalho de caráter provisório, excepcional ou sistemático na sociedade contemporânea.

A continuidade das investigações sobre home-office torna-se importante para analisar outras especificidades da produção científica da ALC e das diversas regiões do mundo. A pesquisa pode ser também relevante para os campos da educação, trabalho e saúde, buscando ampliar o debate sobre o planejamento de políticas públicas nesses domínios. Por meio dos textos de agradecimentos inseridos pelos autores nos seus artigos, sugere-se explorar a colaboração "invisível", uma vez que poucas informações são obtidas através de dados de autoria nas Ciências Sociais, onde os níveis de coautoria comumente são baixos, conforme demonstrado nesta pesquisa. Os dados sobre agradecimentos podem também ser utilizados para examinar características da produção científica sobre home-office financiada da ALC, abrindo novos caminhos para identificar particularmente diferentes tipos de financiamento, agências de fomento, grupos, linhas de pesquisa, pesquisadores e estudantes de pós-graduação financiados. Da mesma forma, importa ampliar o olhar sobre a literatura acadêmico-científica sobre home-office no contexto mundial nos próximos anos, retomando a inserção da ALC nesse contexto, de modo a compreender o desenvolvimento e desdobramentos do tema no período da pandemia de Covid-19 e anos posteriores.

\section{AGRADECIMENTOS}

Os autores agradecem aos pareceristas anônimos pelos comentários e sugestões.

\section{REFERÊNCIAS}

ALIAGA, F. M. Evolución de la edición científica en revistas de Ciencias Sociales y determinación de Latinoamérica como ecosistema característico. RELIEVE - Revista Electrónica de Investigación y Evaluación Educativa, Granada, v. 26, n. 2, M1, 2020. Disponível em: https://doi.org/10.7203/relieve.26.2.19206. Acesso em: 15 jun. 2021. 
ALVAREZ, G. R.; VANZ, S. A. de S.; BARBOSA, M. C. B. Scientometric indicators for Brazilian research on High Energy Physics, 1983-2013. Anais da Academia Brasileira de Ciências, Rio de Janeiro, v. 89, n. 3, supl. p. 2525-2543, 2017. Disponível em:

https://doi.org/10.1590/0001-3765201720160620. Acesso em: 20 jul. 2021.

ALVAREZ, G. R.; CAREGNATO, S. E. Colaboração de subautoria: estudo bibliométrico baseado nos artigos brasileiros com agradecimentos na Web of Science. Encontros Bibli: Revista Eletrônica de Biblioteconomia e Ciência da Informação, Florianópolis, v. 26, p. 01-19, 2021. Disponível em: https://periodicos.ufsc.br/index.php/eb/article/view/74605. Acesso em: 07 jul. 2021.

BARROS, A. M.; SILVA, J. R. G. da. Percepções dos indivíduos sobre as consequências do teletrabalho na configuração home-office: estudo de caso na Shell Brasil. Cadernos EBAPE.BR, Rio de Janeiro, v. 8, n. 1, p. 71-91, mar. 2010. Disponível em: https://doi.org/10.1590/S167939512010000100006. Acesso em. 16 ago. 2021.

BRASIL. Ministério da Economia. PNADc - IBGE: Trimestre: abr-jun/20. Brasília, DF: Ministério da Economia, 2020. Disponível em: https://www.gov.br/fazenda/pt-br/centrais-deconteudos/publicacoes/conjuntura-economica/emprego-e-renda/2020/ie-pnadc-junho2020.pdf. Acesso em: 28 abr. 2021.

COLLAZO-REYES, F. Growth of the number of indexed journals of Latin America and the Caribbean: the effect on the impact of each country. Scientometrics, Amsterdam, v. 98, n. 1, p. 197-209, 2014. Disponível em: https://doi.org/10.1007/s11192-013-1036-2. Acesso em: 22 set. 2021.

FETZNER, M. A. de M. A viabilidade do teletrabalho na PROCEMPA. 2001. $211 \mathrm{f}$. Dissertação (Mestrado em Administração) - Programa de Pós-Graduação em Administração, Escola de Administração, Universidade Federal do Rio Grande do Sul, Porto Alegre, 2001. Disponível em: http://hdl.handle.net/10183/3568. Acesso em: 10 ago. 2021.

FILARDI, F.; CASTRO, R. M. P. de; ZANINI, M. T. F. Vantagens e desvantagens do teletrabalho na administração pública: análise das experiências do Serpro e da Receita Federal. Cadernos EBAPE.BR, Rio de Janeiro , v. 18, n. 1, p. 28-46, jan. 2020. Disponível em: https://doi.org/10.1590/1679-395174605. Acesso em: 16 ago. 2021.

FLACH, N. Home office definitivo? Para 74\% das empresas no Brasil, a resposta é sim. Exame, maio 2020. Disponível em: https://exame.com/carreira/home-office-definitivo-para-74-das-empresas-no-brasil-aresposta-e-sim. Acesso em: 28 abr. 2021.

FUNDAÇÃO INSTITUTO DE ADMINISTRAÇÃO (FIA). Pesquisa Gestão de Pessoas na Crise da Covid-19: Relatório Final. São Paulo: FIA, 2020. Disponível em: https://jornal.usp.br/wp-content/uploads/2020/11/Pesquisa-Gest\%C3\%A3o-de-Pessoas-naCrise-de-Covid-19-ITA.pdf. Acesso em: 28 abr. 2021. 
GLÄNZEL, W. Bibliometrics as a research field: a course on theory and application of bibliometric indicators. [s. I.]: [s. n.], 2003.

GOLDSCHMIDT-CLERMONT, L. Communication patterns in high-energy physics. High Energy Physics Libraries Webzine, [s. I.], n. 6, mar. 2002.

GÓMEZ, S. M. et al. Stress and myths related to the Covid-19 pandemic's effects on remote work. Management Research: Journal of the Iberoamerican Academy of Management, v. 18, n. 4, p. 401-420, 2020. Disponível em: https://doi.org/10.1108/MRJIAM-06-2020-1065. Acesso em: 24 ago. 2021.

HARA, N. et al. An emerging view of scientific collaboration: scientists' perspectives on collaboration and factors that impact collaboration. Journal of the American Society for Information Science and Technology, New York, v. 54, n. 10, p. 952-965, 2003. Disponível em: https://doi.org/10.1002/asi.10291. Acesso em: 26 set. 2021.

HAZAN, B. F.; MORATO, A. Teletrabalho no direito brasileiro: fundamentos, perspectivas e alterações promovidas pela reforma trabalhista. Lex Humana, Petrópolis, v. 10, n. 1, p. 1-23, 2018. Disponível em: http://seer.ucp.br/seer/index.php/LexHumana/article/view/1584. Acesso em: 12 ago. 2021.

INSTITUTO BRASILEIRO DE GEOGRAFIA E ESTATÍSTICA (IBGE). PNAD Covid-19. Rio de Janeiro: IBGE, 2020. Disponível em: https://covid19.ibge.gov.br/pnad-covid/trabalho.php. Acesso em: 28 abr. 2021.

KATZ, J. S. Geographical proximity and scientific collaboration. Scientometrics, Amsterdam, v. 31, n. 1, p. 31-43, 1994. Disponível em: https://doi.org/10.1007/BF02018100. Acesso em: 22 set. 2021.

LANA, R. M. et al. Emergência do novo coronavírus (SARS-CoV-2) e o papel de uma vigilância nacional em saúde oportuna e efetiva. Cadernos de Saúde Pública, Rio de Janeiro, v. 36, n. 3, e00019620, 2020. Disponível em: https://doi.org/10.1590/0102-311X00019620. Acesso em: 26 ago. 2021.

LEMOS, A. H. da C.; BARBOSA, A. de O.; MONZATO, Priscila Pinheiro. Women in home office during the Covid-19 pandemic and the work-family conflict configurations. RAE-Revista de Administração de Empresas, São Paulo, v. 60, n. 6, p. 388-399, nov./dez. 2020. Disponível em: https://doi.org/10.1590/S0034-759020200603. Acesso em: 15 ago. 2021.

LIM, Vivien K. G.; TEO, Thompson S. H. To work or not to work at home - An empirical investigation of factors affecting attitudes toward teleworking. Journal of Managerial Psychology, v. 15, n. 6, p. 560-586, 2000. Disponível em: https://doi.org/10.1108/02683940010373392. Acesso em: 21 ago. 2021.

LOW, W. Y. et al. Trend and impact of international collaboration in clinical medicine papers published in Malaysia. Scientometrics, Amsterdam, v. 98, n. 2, p. 1521-1533, 2014. Disponível: https://doi.org/10.1007/s11192-013-1121-6. Acesso em: 26 set. 2021. 
MANE, K. K.; BÖRNER, K. Mapping topics and topic bursts in PNAS. Proceedings of National Academy of Sciences of the United States of America - PNAS, Washington, v. 101, supl. 1, p. 5287-5290, abr. 2004. Disponível em: https://doi.org/10.1073/pnas.0307626100. Acesso em: 22 set. 2021.

MASUDA, A. D.; HOLTSCHLAG, C.; NICKLIN, J. M. Why the availability of telecommuting matters: The effects of telecommuting on engagement via goal pursuit. Career Development International, v. 22, n. 2, p. 200-219, 2017. Disponível em: https://doi.org/10.1108/CDI-052016-0064. Acesso em: 22 ago. 2021.

MENDES, D. C.; HASTENREITER FILHO, Horacio Nelson; TELLECHEA, Justina. A realidade do trabalho home office na atipicidade pandêmica. Revista Valore, Volta Redonda, v. 5, Edição especial, p. 160-191, 2020. Disponível em: https://doi.org/10.22408/reva502020655160191. Acesso em: 16 ago. 2021.

OLIVEIRA, A. B. de. Los libros en Ciencias Sociales y Humanidades en Brasil: un estudio a partir de los investigadores y de las editoriales. 2018. 498 f. Tese (Doutorado em Ciências de la Documentación) - Universidad Complutense de Madrid, 2018. Disponível em: https://eprints.ucm.es/id/eprint/55556/1/T41139.pdf. Acesso em: 19 jul. 2021.

ORGANISATION FOR ECONOMIC CO-OPERATION AND DEVELOPMENT (OECD). Revised Field of Science and Technology (FOS) classification in the Frascati Manual. Paris: OECD, 2007. Disponível em: https://www.oecd.org/science/inno/38235147.pdf. Acesso em: 15 jul. 2021.

RAFALSKI, J. C.; ANDRADE, A. L. de. Home-office: aspectos exploratórios do trabalho a partir de casa. Temas em Psicologia, Ribeirão Preto, v. 23, n. 2, p. 431-441, 2015. Disponível em: https://doi.org/10.9788/TP2015.2-14. Acesso em: 21 ago. 2021.

RÀFOLS, I. et al. On the dominance of quantitative evaluation in 'peripheral'countries: auditing research with technologies of distance. Social Science Research Network - SSRN, p. 1-22, ago. 2016. Disponível em: https://doi.org/10.2139/ssrn.2818335. Acesso em: 28 set. 2021.

RALPH, P. et al. Pandemic programming - How Covid-19 affects software developers and how their organizations can help. Empirical Software Engineering, v. 25, p. 4927-4961, 2020. Disponível em: https://doi.org/10.1007/s10664-020-09875-y. Acesso em: 22 ago. 2021.

RED DE INDICADORES DE CIENCIA Y TECNOLOGÍA IBEROAMERICANA E INTERAMERICANA (RICYT). El Estado de la Ciencia 2020. Buenos Aires: RICYT, 2020. Disponível em: http://www.ricyt.org/wp-content/uploads/2021/02/ElEstadoDeLaCiencia_2020.pdf. Acesso em: 15 jul. 2021.

ROSENFIELD, C. L.; ALVES, D. A. de. Autonomia e trabalho informacional: o teletrabalho. DADOS - Revista de Ciências Sociais, Rio de Janeiro, v. 54, n. 1, p. 207-233, 2011. Disponível em: https://doi.org/10.1590/S0011-52582011000100006. Acesso em: 13 set. 2021. 
ROYAL SOCIETY. Knowledge, networks and nations: global scientific collaboration in the 21st century. London: Royal Society, 2011. Disponível em: https://royalsociety.org/topicspolicy/projects/knowledge-networks-nations/report. Acesso em: 18 ago. 2021.

SANTIN, D. M.; CAREGNATO, S. E. Concentración y desigualdad científica en América Latina y el Caribe a principios del siglo XXI: un estúdio cienciométrico. Información, Cultura y Sociedad, Buenos Aires, n. 43, p. 13-30, dez. 2020. Disponível em:

http://revistascientificas.filo.uba.ar/index.php/ICS/article/view/8131. Acesso em: 15 jul. 2021.

SOLíS, M. S.. Telework: conditions that have a positive and negative impact on the workfamily conflict. Academia Revista Latinoamericana de Administración, v. 29, n. 4, p. 435449, 2016. Disponível em: https://doi.org/10.1108/ARLA-10-2015-0289. Acesso em: 12 ago. 2021.

TESTA, J. The globalization of Web of Science (2005-2010). Thomson Reuters, 2011. Disponível em: http://wokinfo.com/media/pdf/globalwos-essay. Acesso em: 17 maio 2021.

UNITED NATIONS EDUCATIONAL, SCIENTIFIC AND CULTURAL ORGANIZATION - UNESCO. Unesco Science Report 2015: towards 2030. Paris: Unesco, 2015.

UNITED NATIONS. Statistics Division. Standard Country or Area Codes for Statistics Use. 2021. Disponível em: https://unstats.un.org/unsd/methodology/m49. Acesso em: 15 jul. 2021.

VANZ, S. A. de S. As redes de colaboração científica no Brasil: 2004-2006. 2009. 204 f. Tese (Doutorado em Comunicação e Informação). Universidade Federal do Rio Grande do Sul, Porto Alegre, 2009. Disponível em: http://hdl.handle.net/10183/17169. Acesso em: 19 jul. 2021.

VÉLEZ-CUARTAS, G. J.; LUCIO-ARIAS, D.; LEYDESDORFF, L. Regional and global science: Publications from Latin America and the Caribbean in the SciELO Citation Index and the Web of Science. El profesional de la Información, Barcelona, v. 25, n. 1, p. 35-46, jan./fev. 2016. Disponível em: https://doi.org/10.3145/epi.2016.ene.05. Acesso em: 15 set. 2021.

VENEGAS TRESIERRA, C. E.; LEYVA POZO, A. C. La fatiga y la carga mental en los teletrabajadores: a propósito del distanciamiento social. Revista Española de Salud Pública, Madrid, v. 94, n. 9, p. 1-17, 2020. Disponível em:

https://pesquisa.bvsalud.org/portal/resource/esSiqueira/ibc-199998. Acesso em: 26 ago. 2021.

VOLINO-SOUZA, M. et al. Covid-19 quarantine: impact of lifestyle behaviors changes on endothelial function and possible protective effect of beetroot juice. Frontiers in Nutrition, v. 7, P. 1-7, out. 2020. Disponível em: https://doi.org/10.3389/fnut.2020.582210. Acesso em: 22 ago. 2021. 